\title{
It Just Takes One Phone Call: Simultaneous Activation to Reduce Referral Times
}

Fatimah Lateef, FRCS (A\&E), MBBS, FAMS (Em Med)

Senior Consultant, Dept of Emergency Medicine, Singapore General Hospital, Professor, Duke NUS Graduate Medical School, Professor, Yong Loo Lin School of Medicine, National University of Singapore, Director, SingHealth Duke NUS Institute of Medical Simulation (SIMS).

*Corresponding Author: Fatimah Lateef, Senior Consultant, Dept of Emergency Medicine, Singapore General Hospital, Professor, Duke NUS Graduate Medical School, Professor, Yong Loo Lin School of Medicine, National University of Singapore, Director, SingHealth Duke NUS Institute of Medical Simulation (SIMS).

Received date: July 21, 2020; Accepted date: September 04, 2020; Published date: September 11, 2020.

Citation: F Lateef. (2020) It Just Takes One Phone Call: Simultaneous Activation to Reduce Referral Times. Clinical Orthopaedics and Trauma Care. 2(2); DOI:10.31579/2694-0248/009

Copyright:@2020 Fatimah Lateef, This is an open-access article distributed under the terms of the Creative Commons Attribution License, which permits unrestricted use, distribution, and reproduction in any medium, provided the original author and source are credited.

\begin{abstract}
When there are maternal and neonatal emergencies in the Emergency Department, Emergency Physicians will manage them and will call on the Obstetrics and Gynaecology as well as Neonatalogy doctors and staff as needed. Thus far, these staff have to call individually and it takes time to repeat the history and relevant information to each one of them. We embarked on an initiative to have simultaneous activation of the relevant personnel once such emergencies surfaced. This would be through an agreed upon model and will be conducted via the operator and institution's switchboard. All the relevant staff involved in the activation will get the information straight away, simultaneously and immediately upon activation.

To be able to do this, we organised several meetings and brainstorming sessions with the disciplines involved, tried out insitu simulation scenarios with failure mode effect analysis, as well as applied the PDSA cycle (Plan, Do, Study and Act) to the initiative. After the robust testing and discussions, which took some 6 months, the final pathway and algorithm for simultaneous activation and management was executed.
\end{abstract}

Key words: maternal emergencies; emergency department; simultaneous activation; insitu simulation; failure mode effect analysis

\section{Introduction and Background}

Singapore General Hospital is a tertiary referral centre and teaching institution. It is part of an Academic Medical Centre, together with Duke NUS Graduate Medical School on the same campus. There are 1800 beds spanning across 36 different specialties. The Emergency Department tends to some 140000 patients annually. The institution also has a renown Department of Obstetric and Gynaecology (OG) and a Department of Neonatology, including a Neonatal Intensive Care Unit. When there are maternal emergencies in the $\mathrm{ED}$, frontline emergency physicians will manage them and can contact the OG doctors by calling the on-call doctor. The same goes for the neonatologist on call. The maternal emergencies would include maternal cardiac arrest, eclamptic seizures, antenatal and postnatal (post-partum haemorrhage) obstetric haemorrhage. At times, coupled with the maternal emergencies are obstetric emergencies such as shoulder dystocia, breech delivery, premature baby delivery and cord prolapse. For all these, the on call OG doctors would be contacted together with the neonatology registrar. [1,2] When the emergency is one which is likely to need surgery in the emergency operating theatre (eg. cord prolapse, massive post-partum haemorrhage), the anesthesiologist on call as well as the surgical intensive care unit (ICU) registrar would also have to be called, individually.

The time taken for these personnel to arrive at the ED resuscitation room varies due to a variety of factors, including how fast they could be contacted by the ED staff. Over the years, there have been feedback on some challenges and difficulties in the micro-system described above. These could be related to time delays, people/human factors, equipment issues and lack of clear workflow processes.[3] When there is no response based on the first phone call, the staff will have to try again until a response is obtained with confirmation to come down to the ED. During the phone-call, the staff who is calling would have to summarize the gist of the case and this would of course, take some time as well. This would have to be repeated for every person who is being contacted from the various disciplines involved in the care.

As a result of this, it was decided that a review and brainstorming of the work flow and processes was needed. This would be conducted with an inter-professional team, from all the relevant disciplines. A simple, clear and effective workflow would be desirable. Every discipline must be clear about their roles, responsibilities and commitment as members of the team. [3]

From the review, a consolidated algorithm was generated based on the agreement and inputs from all disciplines. This was attained after several rounds of brainstorming to ensure the needs and requests are all looked into.

Insitu Simulation and Healthcare Failure Mode Analysis 
Our team decided that an insitu simulation would be one of the best ways to assess the algorithm, work processes and identify latent threats. In-situ simulation (ISS) is defined as Point Of Care simulation taking place in the actual clinical environment during the working shifts. It can be used to test overall preparedness and seamlessness with a particular workflow and/or a process. It promotes experiential learning, which is closely aligned to actual work experiences. [4-6]

The scenarios for the ISS were carefully selected to cover the range of maternal, obstetrics and neonatal emergencies in various combinations, to test and stress the workflow and micro-systems in place. These insitu simulation scenarios, usually commenced in the ED resuscitation room and will follow through into the labour ward, admitting ward or the emergency operating theatre, as appropriate [7, 8]. Some examples of the scenarios include, maternal cardiac arrest, premature labour, breech deliver, cord prolapse and eclamptic seizures.

In general, certain obstetric/ maternal emergencies are rare. The potential for legal liabilities makes dependence on learning at the bedside with real life events impractical and a potential threat to patient safety. Simulation

is thus a viable alternative teaching strategy, both for learning new skills as well as maintaining these skills. Simulation will help prepare the team for the high risk and relatively rare events. The simulation also offers a safe environment to practice and make mistakes, learn from them and take the necessary corrective actions, in order to improve work flow processes and skills. [9]

In order to make this exercise as comprehensive as possible, it was decided to combine ISS with the concept of Healthcare Failure Mode Effect Analysis (FMEA). The Failure Mode and Effects Analysis (FMEA) model in healthcare is a quality improvement tool which usually involves an inter-professional team assembled to identify systemic risks, using the steps as shown in (Table 1). [10, 11] FMEA is used prospectively to identify systemic failures which need to be addressed, with the overall system made more robust as a result. FMEA focuses on processes and not on a specific event. FMEA helps to pinpoint failure modes derived from expert opinion and statistical estimates, as opposed to an evaluation of a specific process under actual operating conditions. Thus, our plan to combine both ISS with FMEA was based on our goal of making the experience as practically oriented as possible. The drill utilizing ISS and FMEA will assist to ensure a more comprehensive and wholesome look at the processes, operations and logistics. It also helps to ensure that no tasks are redundant or omitted [10-12].

\begin{tabular}{|c|c|c|}
\hline Phases & In situ Simulation & FMEA \\
\hline Identification of Processes & $\begin{array}{l}\text { Identify critical and time-dependent } \\
\text { processes and tasks }\end{array}$ & $\begin{array}{l}\text { Breakdown of processes and tasks into } \\
\text { small or component steps }\end{array}$ \\
\hline Allocation of Teams & $\begin{array}{l}\text { Simulation team performers: from our } \\
\text { modular ED teams to prevent cross } \\
\text { interaction and mixing and including the } \\
\text { team members from OG, Neonatology, } \\
\text { Nursing etc }\end{array}$ & $\begin{array}{l}\text { Team made up of senior faculty, nursing } \\
\text { managers, modular team leaders, senior } \\
\text { resident physician and senior staff nurse. } \\
\text { They will make observations from the } \\
\text { simulation, identify gaps, lapses and } \\
\text { failure modes needing corrective action } \\
\text { and follow-up }\end{array}$ \\
\hline Description of Processes & $\begin{array}{l}\text { Process mapping of management in } \\
\text { detail, with time considerations }\end{array}$ & $\begin{array}{l}\text { Testing of efficiency and adequacy of } \\
\text { algorithms and flowcharts. Also what } \\
\text { changes need to be implemented post } \\
\text { session/ drill }\end{array}$ \\
\hline List of Observations & $\begin{array}{l}\text { Gaps, lapses etc needing change and } \\
\text { corrective action identified. Also } \\
\text { collected from debrief session after the } \\
\text { simulation }\end{array}$ & $\begin{array}{l}\text { List all failure modes ( based on the } \\
\text { experiences of the team members and the } \\
\text { expert panel doing the observations) }\end{array}$ \\
\hline Root Cause Analysis (RCA) & $\begin{array}{l}\text { The RCA is a common session with } \\
\text { FMEA }\end{array}$ & The RCA is a common session with ISS \\
\hline Implementation and Follow up Action & $\begin{array}{l}\text { Change and update algorithm and work } \\
\text { processes/ Ensure implementation of } \\
\text { these changes and updates. Also to } \\
\text { communicate these to the whole } \\
\text { department in a timely fashion ( email } \\
\text { blast, private chat groups, flyers and } \\
\text { charts can be put up in the department and } \\
\text { work areas) }\end{array}$ & $\begin{array}{l}\text { Change and update algorithm and work } \\
\text { processes/ Ensure implementation of these } \\
\text { changes and updates. Also to } \\
\text { communicate these to the whole } \\
\text { department in a timely fashion ( email } \\
\text { blast, private chat groups, flyers and charts } \\
\text { can be put up in the department and work } \\
\text { areas) }\end{array}$ \\
\hline
\end{tabular}

Table 1: Combining In situ simulation and HealthCare Failure Mode Effect and Analysis (FMEA)

Active participation from the inter-professional teams during the ISSFMEA before the actual implementation will facilitate the evaluation of critical points and the subsequent changes before the final application. These preliminary steps can help in the assessment of adequacy of the structure as well as prepare the staff from different departments for better compliance with the algorithm and protocols when implementation commences $[3,12]$.

In taking this approach, it may take a longer time to implement a new or updated protocol, but we feel this is a robust and unified approach. Moreover, it gives the inter-professional team a coordinated way to execute care, which is adequately researched and is evidence-based. The 
training conducted helped build and strengthen inter-professional relationships, as well as enhance the competencies for maternal and neonatal care. [13-16] Simulation-based training using ISS reinforces evidence-based practices, whilst improving communications and teamwork skills under realistic ED conditions $[5,9]$.

\section{The Plan-Do-Study-(ReDo)-Act Cycle}

Our model of approach for the proposed change is using the Plan-DoStudy-Act Cycle (PDSA Cycle). This is a quality improvement methodology and tool which we felt can be readily and easily applied to our context in healthcare to help embrace and support our intent. Afterall, we want to have a delivery of care which is timely, safe, efficient and effective for our patients. The PDSA tool is relevant for us as it focuses on the crux of change and translation of ideas from our discussions and brainstorming session into executable actions. It would also enable us to incorporate new practices from our learning during ISS/ FMEA drills into the new model proposed. [17, 18] As healthcare is a complex environment, we felt there was sophistication in the simplicity of the PDSA model and extremely practical to apply. The model helped in our formulation of the hypothesis, collecting information and data as needed, analyzing and interpreting information and observations we had and finally, making the right inferences to execute the new, changed model of work $[17,19]$.

\section{Steps involved in our PDSA Cycle}

Plan:

1. Conceptualization of idea/ intent and exploration through factfinding

2. Gather (inter-professional) personnel as relevant to change

3. Meetings or virtual meeting and brainstorming sessions. Numbers can vary according to needs.

4. Listening, discussion with contributions from all relevant parties

5. Exploring pros and cons, strengths and weaknesses of the current processes if any

6. Coming forth with drafts. There may be several drafts as changes are being made

7. For some interventions, team members may have to conduct site visits to understand the situation

8. Review of preparation, equipment, arrangements etc as relevant

9. Initial literature search

Do:

10. Appoint champions and leaders as needed

1. Plan and prepare for the ISS/ FMEA

2. Write scenarios to test as appropriate and get concurrence of the IP teams

3. Conduct the ISS/ FMEA: may have several rounds, using different scenarios, may have to conduct during the different shifts in the ED

Study:

1. Much information will be obtained from debrief and feedback sessions following the drill using ISS/ FMEA

2. Recognize inputs from participants as well as inter-professional observers carrying out the FMEA

3. Address chaos and confusion which may have arisen

4. Explore the latent threats observed if any and seek for solutions/ resolution

5. Explore options and alternatives suggested from participants and check against your objectives and proposed workflow processes
6. Conduct root cause analysis as needed

7. Review if need to appoint more champions and leaders at different levels and from different disciplines

8. Edit algorithm and work flow

9. May have to "Re-Do" ie conduct another ISS/ PMEA to use the updated and edited model workflow.

Act:

10. Finalise algorithm and workflow

1. Publicize the new model. Get help from the appointed champions

2. Use posters, email blasts, publicity flyers to update everyone

3. In some cases a road show may be relevant. A "Question and Answer" session may be relevant in some cases

4. Commence execution of the new workflow process

5. Monitor closely as the new workflow is used, in case there are other minor tweaking needed or some 'blind spots' were not addressed. Be prepared to change if needed

\section{Administrative Issues and Technical Testing}

With finalization of the algorithm and workflow, a few other things needed to be sorted out. It was decided that the initiator of the flow, within the institution will be:

\section{Staff from the ED \\ 2. Staff from labour ward \\ 3. Staff from OG wards}

Any of the above staff will only need to pick up the telephone and dial " 0 " for the operator at switchboard. They then need to state clearly the relevant information. The message must include a one statement summary of the case and also the exact location. It is not good enough to state: "35 year old Gravida 1, Para 0, with cord prolapse at the ED". It is better to state, "35 year old Gravida 1, Para 1, with cord prolapse at the ED Resus Room Panel 2".

All the inter-professional personnel involved will be activated simultaneously by the operator and they will receive the message all at the same time on their mobile devices, registered with the institution.(2022) Once the message is received they will then make their way promptly to the mentioned location [3].

All activation cases will be audited. Forms, either physical or electronic, will have to be completed.

\section{Discussion}

Hospitals represent complex, multi-faceted systems, with subdivisions into macro- and micro-systems. It is important to ensure well-coordinated services as well as continuity of essential services as patients move from one area to the next within the institution. There must be timely handling and swift adaptation to the changing needs and demands. This is especially so with critically ill patients, with time-dependent conditions. Planning and training will help to ensure effective use of resources, familiarity with workflow processes and efficient involvement of the right specialists at the right time [23]. Planning this simultaneous activation code took some 6 months, from the conceptualization phase, to setting the clear achievable objectives, meetings with relevant inter-professional colleagues, coordinating the ISS/ FMEA, reviewing and testing out the codes and fine-tuning the final algorithm to meet every discipline's needs. (Table 2) All these steps are important to ensure we start off this code activation on a positive note, with buy-in from all parties. We plan to conduct debrief after each actual code activation and use this platform for a quality improvement project and intervention. 


\section{SGH ObstetCode Activation}

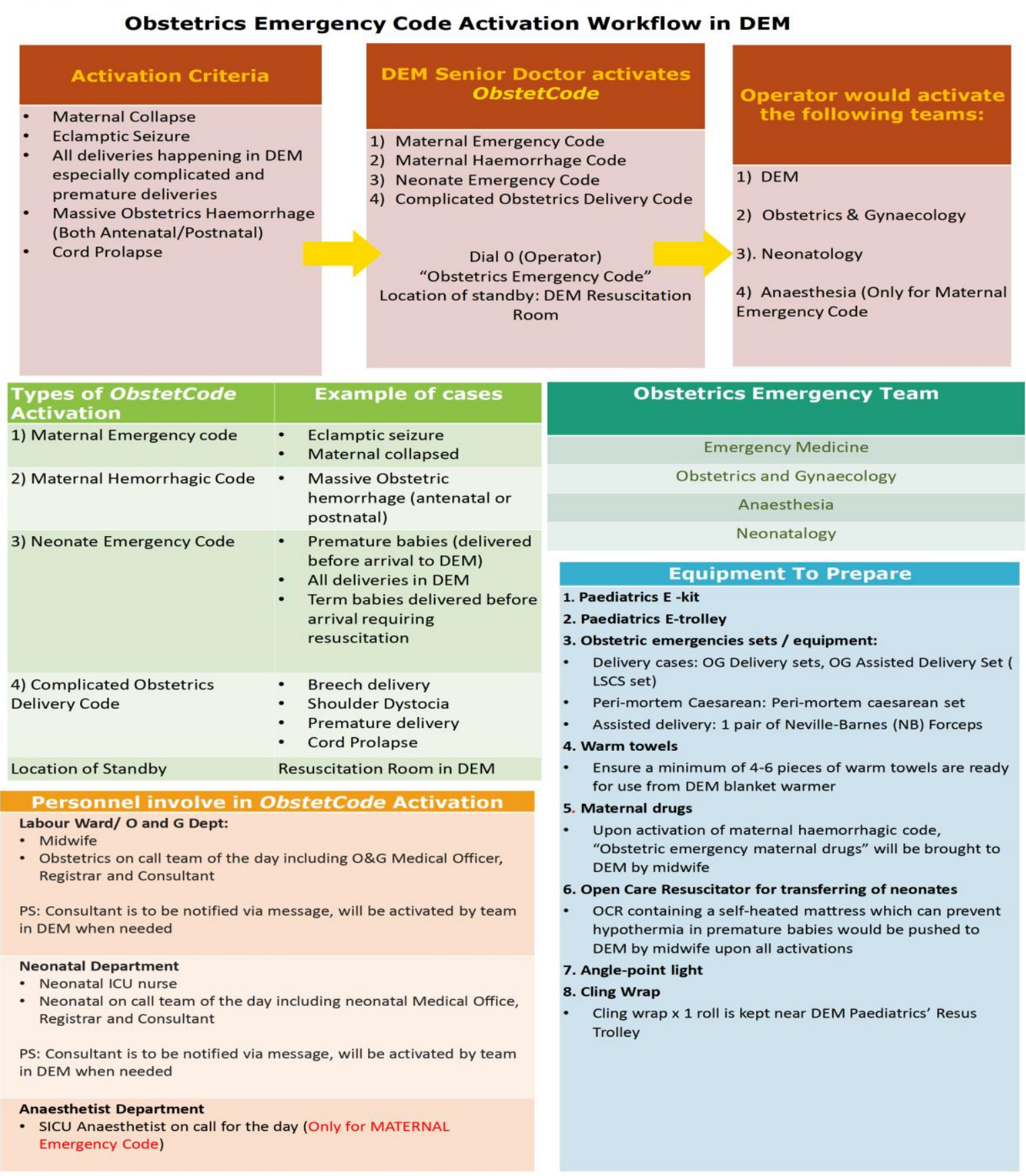

Table 2: The sample ObstetCode Activation Algorithm

\section{Conclusion}

An innovative and collaborative approach to obstetric/ neonatal emergencies can make these episodes more manageable, in a timely fashion, with enhanced levels of patient safety and better outcomes. One of the key changes is our replacement of the sequential activation and calling process with the simultaneous activation of the team. This is indeed a major paradigm shift and a necessary one. Inter-professional team responses such as this must proceed in a coordinated and choreographed fashion to ensure efficiency and effectiveness in execution.

\section{References}

1. Draycott T, Sibanda T, Owen L, Akande V, Winter C, Reading S et al. (2006) Does training in obstetric emergencies improve neonatal outcomes. BJOG. 113(2): 177-182.

2. Hilton G, Daniels K, Goldhaber-Fiebert SN, Lipman S, Carvallo B, Butwick A. (2016) Checklists and multidisciplinary team performance during simulated obstetric haemorrhage. Int $\mathbf{J}$ Obstet Anaesth; 25: 9-16.

3. Lateef F. (2018) Inter-professional education, inter-professional practice and team science: learning together, working together. Edu Med Journal. 10: 81-91. 
4. Hansen SS, Arafeh J. (2012) Implementing and sustaining insitu drills to improve multi-disciplinary healthcare training. Journal of Obstetrics, Gynecologic and Neonatal Nursing. 41(4): 559-571.

5. Crofts JF, Winter C, Souter MC. (2011) Practical simulation training for maternity care: where we are and where next? BJOG; 118(Suppl 3): 11-16.

6. Lateef F. (2020) Enhancing the satisfaction and learning experience of Year 4 medical students with the addition of high fidelity simulation scenario in their EM posting. Advances in Med and Clin Sciences Res. 1: 1-6.

7. Goldshtein D, Krensky C, Doshi S, Perelman VS. (2020) Insitu simulation and its effect on patient outcomes: A systematic review. BMJ Simulation and Technology Enhanced Learning. 6: 3-9.

8. Schofield C, Welfare E, Mercer S. (2018) Insitu simulation. Trauma. 20(4): 281-288.

9. Lateef F. (2010) Simulation-based learning: Just like the real thing. Journal of Emergency, Trauma and Shock. 3: 348-352.

10. Faierla G, Paranel A, Franklin BD, Chana P, Cesarelli M, Stanton NA, Serdalis N. (2018) Expanding healthcare failure mode effect analysis approach. Reliability Engineering and Systems Safety. 169: 117-126.

11. De Vries M, Fan M, Tscheng D, Hamilton M, Trbovich P. (2019) Clinical observations and a healthcare failure mode effect analysis to identify vulnerabilities in the security and accounting of medications in Ontario hospitals: A study protocol. BMJ Open. 9: e027629.

12. Al Kadri HMF. (2010) Obstetric medical emergency teams are a step forwards in maternal safety. Journal of Emergency, Trauma and Shock. 3(4): 337-341.
13. Katz VL. (2012) Perimortem caesarean delivery. Its role in maternal mortality. Semin perinatal. 36: 68-72.

14. Marshall S. (2013) The use of cognitive aids during emergencies in anaesthesia: A review of the literature. Int Anaesth Analg. 117: 1162-1171.

15. Wheatley S. (2010) Maternal Critical care. Whats in a name? Int $\mathbf{J}$ Obs Anesth. 19(4): 353-355.

16. Verceuil A, Hopkins P. (2015) Maternal Critical care. A time to embrace continuity. Int J of Obs Anesth. 24(30: 203-206.

17. Reed JE, Card AJ. (2016) The problem with PDSA cycles. BMJ Quality and safety.25: 147-152.

18. PDSA cycles and the model for improvement. ACT Academy.

19. Coury J, Schreider JL, Rivelli JS, Petrik A, Seibel L, D' Agostini B et al. (2017) Applying the PDSA cycle to a large pragmatic study involving safety net clinics. BMC health Serv Res.

20. Fatimah Lateef. (2018) Clinical reasoning: the core of medical education and practice. Int J Intern and Emerg Med. 1(2): Article 1015.

21. Jeejeebhoy FM, Zelop CM, Lipman S, Carvalho B, Joglar J, Mhyre $\mathrm{J}$ et al. (2015) cardiac arrest in pregnancy. A scientific statement from the American Heart Association. Circulation. 132(18): 17471773.

22. Winters B, Pham J, Pronovost P. (2006) Rapid response teams walk. Don't run. JAMA. 296: 1645-1647.

23. King E, Wowath R, Shulken DJ. (2006) Establishing a rapid response team in an academic hospital: One years' experience. J Hosp Med. 1: 296-305. 